\title{
THE DIVERSITY OF MACROMYCETES IN THE TERRITORY OF BATOČINA (SERBIA)
}

\author{
Nevena N. Petrović*, Marijana M. Kosanić and Branislav R. Ranković \\ University of Kragujevac, Faculty of Science, Department of Biology and Ecology \\ St. Radoje Domanović 12, 34000 Kragujevac, Republic of Serbia \\ *Corresponding author; E-mail: nevena.n.petrovic@pmf.kg.ac.rs
}

(Received March 29th, 2019; Accepted April 30th, 2019)

\begin{abstract}
The purpose of this paper was discovering the diversity of macromycetes in the territory of Batočina (Serbia). Field studies, which lasted more than a year, revealed the presence of 200 species of macromycetes. The identified species belong to phyla Basidiomycota (191 species) and Ascomycota (9 species). The biggest number of registered species (100 species) was from the order Agaricales. Among the identified species was one strictly protected - Phallus hadriani and seven protected species: Amanita caesarea, Marasmius oreades, Cantharellus cibarius, Craterellus cornucopiaodes, Tuber aestivum, Russula cyanoxantha and $R$. virescens; also, several rare and endangered species of Serbia. This paper is a contribution to the knowledge of the diversity of macromycetes not only in the territory of Batočina, but in Serbia, in general.
\end{abstract}

Keywords: Ascomycota, Basidiomycota, Batočina, the diversity of macromycetes.

\section{INTRODUCTION}

Fungi represent one of the most diverse and widespread group of organisms in terrestrial ecosystems, but, despite that fact, their diversity remains highly unexplored. Until recently it was considered that there are 1.6 million species of fungi, from which only something around 100000 were described (KIRK et al., 2001), while data from 2017 lists 120000 identified species, which is still a slight number (HAWKSWORTH and LÜCKING, 2017). Modern estimations, based on new molecular methods (such as HTS method - highthroughoutput screening) suggest the existence of 5.1 million species of fungi (BLACKWELL, 2011). The number of species of macromycetes was estimated to be 140000 , while the rest of those 1.6 million species belonged to micromycetes which is just 10\% (STAJIĆ, 2015).

Studies of biota in the territory of Batočina remain scarce (GLAVENDEKIC and KOlAROV, 1994; RANKOVIĆ, 2002; MARKOVIĆ et al., 2015) and macromycetes are unexplored. In the territory of Serbia 1300 species and subspecies of macromycetes were noted, but it is estimated that that number is much bigger, somewhere between 3000 and 6 000 species of macromycetes (SADIKOVIĆ and KUŠTERA, 2013). Lack of the national database of fungi and the small number of scientific publications led to poor knowledge about the distribution of macromycetes in Serbia (LAČKOVIĆ, 2015). Even though studying macromycetes in Serbia has been intensified over the past few years (VuKOJEVIĆ and HADŽIĆ, 2013; LUKIĆ, 2013; IVANČEVIĆ, 2016; SAVIĆ, 2016; VuKOJEVIĆ et al., 2016), the diversity of 
macromycetes of Batočina hasn't been studied yet, so the primary aim of this study was the contribution to the knowledge of the diversity of macromycetes in the territory of Batočina.

\section{MATERIALS AND METHODS}

\section{The description of the studied area}

Batočina is a municipality in the central part of Serbia, located in the lower part of the basin of Lepenica river. The municipality is located at a longitude of $21^{\circ} 04^{\prime} \mathrm{E}$ and latitude of $44^{\circ}$ 09' $\mathrm{N}$ and contains ten villages (Brzan, Dobrovodica, Badnjevac, Gradac, Žirovnica, Kijevo, Crni Kao, Milatovac, Nikšić and Prnjavor) and the town of Batočina. It covers the area of $136 \mathrm{~km}^{2}$, from which 10474 ha is agricultural land and 2735 ha is forest area (ILIĆ, 2014). The area is plain and hilly, with average altitude of $110 \mathrm{~m}$. The climate is humid continental, characterized by hot summers, cold winters, with autumn being the warmer period than spring. Precipitation is the greatest during late autumn and late spring. Cambisol, vertisol and alluvial soils are the most common types of soil. Besides river Lepenica, there is a lot of springs, but they are low water springs (ILIĆ, 2014).

The vegetation of the area is represented with plant communities, where different species of oak (Quercus spp.) are dominant and other species can be found, too, such as: common ash (Fraxinus excelsior L.), silver linden (Tilia tomentosa Moench.), common hornbeam (Carpinus betulus L.), hop-hornbeam (Ostrya carpinifolia Scop.) etc. At some localities black pine (Pinus nigra L.) and Scots pine (P. sylvestris L.) can be found. Willow (Salix spp.) and poplar (Populus spp.) forests are present by the river and in moist areas. Black locust forests (Robinia pseudoacacia L.) occur as secondary vegetation in places, where the primary forests had been cut down. Deforestation, degradation and fragmentation of natural habitats are a big problem in the territory of Batočina.

\section{Collecting samples and identification}

Monitoring of fungi in the area of Batočina (Fig. 1) was performed from June 2017 to August 2018. Field studies were conducted in the town of Batočina and several villages: Brzan, Kijevo, Gradac, Prnjavor, Crni Kao and Dobrovodica. Most of the field studies was performed in the town of Batočina (town of Batočina will be referred to as Batočina further in the text). Several localities were investigated in the territory of Batočina, such as: Gornja Batočina, Rogot, Straževica hill, Donja Mala and Gornja Mala. Among the investigated localities, Straževica hill was the locality with the highest altitude $(358 \mathrm{~m})$, while Rogot forest was the only protected locality, representing a natural monument (http://www.zzps.rs/novo/ kontent/stranicy/zastita_prirode_spomenici_prirode/PrirodniSpomenik.pdf).

The localities were visited by a certain dynamic, but more frequently in optimal periods for the growth of macromycetes. Thus, greater number of field trips was in 2018 due to higher rainfall which was favorable for the development of mushrooms' fruiting bodies.

All collected fruiting bodies were photographed using the camera (Canon ixus 185), from all angles, so that the most important taxonomic characteristics could be noted. Photographs were taken in the locality where the basidiocarps were found, unless the circumstances were not proper, in that case photographs of them were taken on a neutral background in the laboratory. For each identified species the locality, date of collection, type of habitat and general conditions of the environment were noted. Fruiting bodies samples were collected manually (usually one mature fruiting body was taken) with a piece of the substrate. 


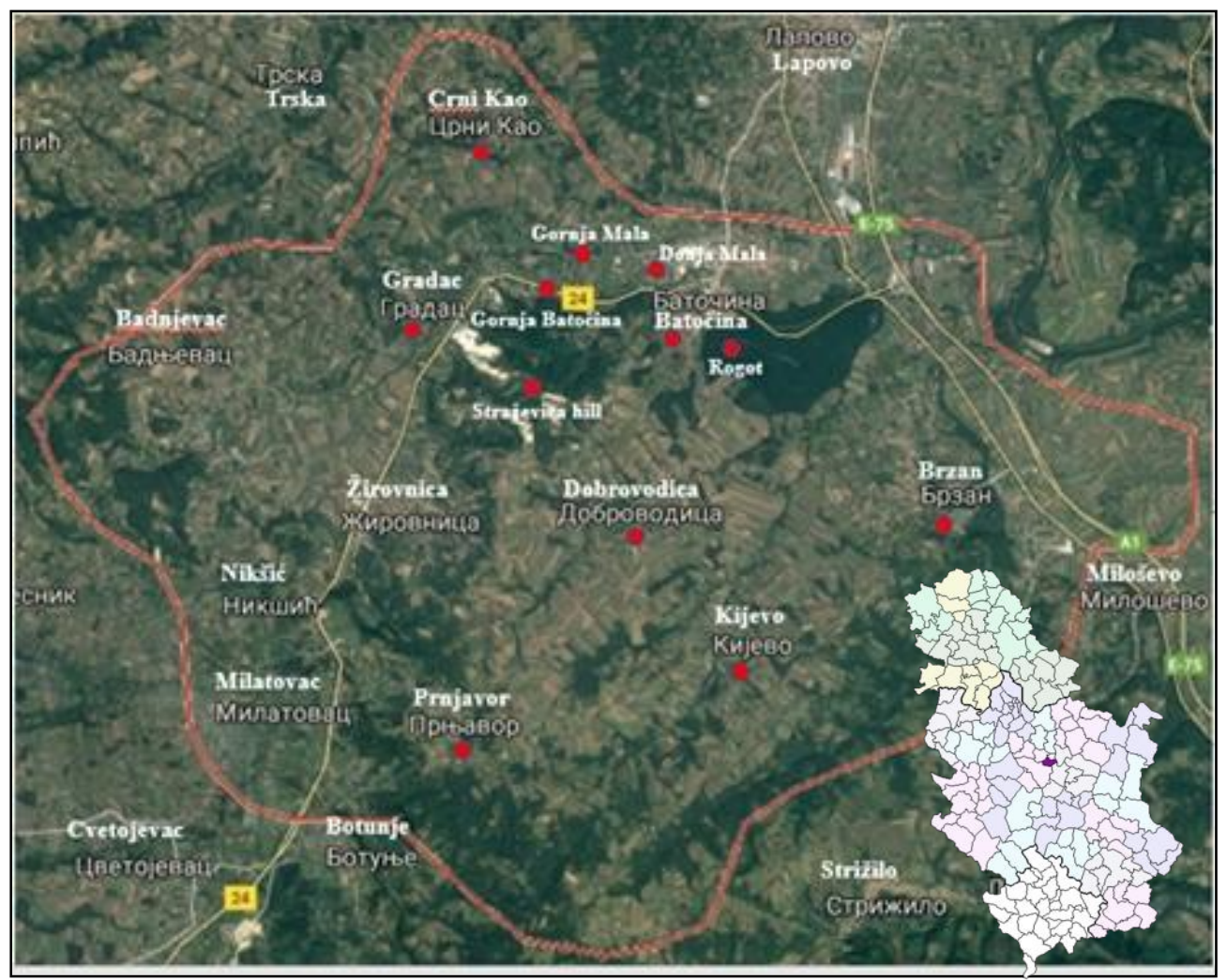

Figure 1. The map of Batočina municipality and its position in the map of Serbia.

The investigated localities are marked with red dots.

(The map of Batočina: https://www.google.com/maps/place/ Баточина/; The map of Serbia: https://upload.wikimedia.org/wikipedia/commons/thumb/b/b3/Serbia_Bato\% C4\%8Dina.png/250pxSerbia_Bato\%C4\%8Dina.png)

Morphological characteristics of the fruiting bodies were firstly analyzed: the type, shape, color, consistency, type of hymenophore, as well as the cross section of the fruiting body, scent and taste (not with every species). In cases where that wasn't sufficient to identify the species, spore print was taken, and the characteristics of spores were observed by Optika B-180 microscope. Some of the identified specimens are preserved in the Department of Biology and Ecology, Faculty of Science, University of Kragujevac.

Specimen identification was done using relevant literature and identification keys (MOSER, 1983; BOŽAC, 1984; FOCHT, 1990; https://nature.berkeley.edu/brunslab/ev/CHLOROPH YLLUM.pdf; UZELAC, 2009; HADŽIĆ, 2012; FLIK, 2017; http://www.vielepilze.de/coprinus/cop key/ecopkey.pdf). The species names were given based on Index Fungorum (http://www.indexfungorum.org/names/names.asp). The identified species were ranked taxonomically, in compliance with the mentioned site. Within taxa, the list of species was presented in alphabetic order.

\section{RESULTS AND DISCUSSION}

The presence of 200 species of macromycetes was recorded in the territory of Batočina municipality. The identified species belong to phyla Basidiomycota (191 species) and Ascomycota (9 species). Species of Basidiomycota phylum were classified into 10 orders and 40 families, while species of Ascomycota phylum were classified into three orders and seven families. The highest number of species belongs to the order Agaricales. The list of the identified species is shown in Table 1. 
Table 1. The list of the identified species of macromycetes in the territory of Batočina municipality.

$\mathrm{SH}=$ Straževica hill; $\mathrm{R}=$ Rogot; $\mathrm{DM}=$ Donja Mala; GM = Gornja Mala; GB = Gornja Batočina; BA = Batočina (the town, urban areas where macromycetes were found - lawns, gardens, by the road, etc.); $\mathrm{K}=$ Kijevo; $\mathrm{D}=$ Dobrovodica; $\mathrm{P}=$ Prnjavor; $\mathrm{G}=$ Gradac; $\mathrm{CK}=\mathrm{Crni}$ Kao. * $=$ strictly protected species, ** $=$ protected species.

\begin{tabular}{|c|c|c|c|}
\hline Species & Family & Order & Date and place of collection \\
\hline \multicolumn{4}{|l|}{ BASIDIOMYCOTA } \\
\hline Agaricus bisporus (J.E. Lange) Imbach & Agaricaceae & Agaricales & 11. 11. 2017 GB \\
\hline Agaricus bohusii Bon. & Agaricaceae & Agaricales & 21. 07. $2018 \mathrm{R}$ \\
\hline Agaricus bresadolanus Bohus & Agaricaceae & Agaricales & 11. 10. $2017 \mathrm{G}$ \\
\hline Agaricus silvicola (Vittad.) Peck. & Agaricaceae & Agaricales & 05. 07. $2018 \mathrm{CK}$ \\
\hline Agaricus xanthodermus Genev. & Agaricaceae & Agaricales & 26. 05. $2018 \mathrm{~GB}$ \\
\hline Calvatia gigantea (Batsch) Lloyd & Agaricaceae & Agaricales & 02. 08. $2018 \mathrm{DM}$ \\
\hline Chlorophyllum rhacodes (Vittad.) Vellinga & Agaricaceae & Agaricales & 05. 07. $2018 \mathrm{CK}$ \\
\hline Chlorophyllum venenatum (Bon) & Agaricaceae & Agaricales & 11. 11. $2017 \mathrm{~GB}$ \\
\hline Cyathus striatus (Huds.) Willd. & Agaricaceae & Agaricales & 21. 07. $2018 \mathrm{R}$ \\
\hline Lepiota clypeolaria (Bull.) P. Kumm. & Agaricaceae & Agaricales & 10. 07. $2018 \mathrm{SH}$ \\
\hline Lepiota cristata (Bolton) P. Kumm. & Agaricaceae & Agaricales & 25. 09. $2017 \mathrm{R}$ \\
\hline Leucoagaricus americanus (Peck) Vellinga & Agaricaceae & Agaricales & 28. 07. $2018 \mathrm{BA}$ \\
\hline Leucoagaricus barssii Zeller (Vellinga) & Agaricaceae & Agaricales & 25. 10. $2017 \mathrm{DM}$ \\
\hline Leucoagaricus leucothites (Vittad.) Wasser & Agaricaceae & Agaricales & 20. 09. $2017 \mathrm{BA}$ \\
\hline Lycoperdon molle Pers. & Agaricaceae & Agaricales & 05. 07. $2018 \mathrm{CK}$ \\
\hline Lycoperdon perlatum Pers. & Agaricaceae & Agaricales & 05. 07. $2018 \mathrm{CK}, \mathrm{SH}$ \\
\hline Lycoperdon pratense Pers. & Agaricaceae & Agaricales & 10. 07. $2018 \mathrm{SH}$ \\
\hline Macrolepiota fuliginosa (Barla) Bon & Agaricaceae & Agaricales & 05. 07. $2018 \mathrm{CK}$ \\
\hline Macrolepiota mastoidea (Fr.) Singer & Agaricaceae & Agaricales & 25. 09. $2017 \mathrm{DM}$ \\
\hline Amanita caesarea (Scop.) Pers. ** & Amanitaceae & Agaricales & 10. 07. $2018 \mathrm{SH}$ \\
\hline Amanita citrina Pers. & Amanitaceae & Agaricales & 22. 07. $2018 \mathrm{SH}$ \\
\hline
\end{tabular}


Table 1. continued

\begin{tabular}{|c|c|c|c|}
\hline Species & Family & Order & Date and place of collection \\
\hline Amanita dryophila Consiglio \& Contu & Amanitaceae & Agaricales & $25.05 .2018 \mathrm{R}$ \\
\hline Amanita pantherina (DC.) Krombh & Amanitaceae & Agaricales & 10. 07. $2018 \mathrm{SH}$ \\
\hline Amanita phalloides (Vaill. ex Fr.) Link & Amanitaceae & Agaricales & 10. 07. $2018 \mathrm{SH}$ \\
\hline Amanita rubescens Pers. & Amanitaceae & Agaricales & 10. 07. $2018 \mathrm{SH}$ \\
\hline Amanita subnudipes (Romagn.) Tullos & Amanitaceae & Agaricales & 25. 05. $2018 \mathrm{R}, \mathrm{SH}$ \\
\hline Amanita vaginata (Bull.) Lam. & Amanitaceae & Agaricales & 25. 05. $2018 \mathrm{R}$ \\
\hline Amanita vaginata var. alba (De Seynes) Gillet & Amanitaceae & Agaricales & 25. 05. $2018 \mathrm{R}$ \\
\hline Conocybe tenera (Schaeff.) Fayod. & Bolbitiaceae & Agaricales & 24. 04. $2018 \mathrm{~GB}$ \\
\hline Coprinellus disseminatus (Pers.) J. E. Lange & Coprinaceae & Agaricales & 11. 11. $2017 \mathrm{~GB}$ \\
\hline Coprinus comatus (D. F. Müll.) Pers. & Coprinaceae & Agaricales & 20. 10. $2017 \mathrm{BA}, \mathrm{G}$ \\
\hline Entoloma clypeatum (L.) P. Kumm. & Entolomataceae & Agaricales & 10. 05. $2017 \mathrm{R}$ \\
\hline Entoloma sepium (Noulet \& Dass.) Richon \& Roze & Entolomataceae & Agaricales & 29. 04. $2018 \mathrm{BA}$ \\
\hline Entoloma sinuatum (Bull.) P. Kumm. & Entolomataceae & Agaricales & 10. 07. $2018 \mathrm{SH}$ \\
\hline Hygrophorus cossus (Sowerby) Fr. & Hygrophoraceae & Agaricales & 29. 09. $2017 \mathrm{DM}$ \\
\hline Hygrophorus eburneus (Bull.) Fr. & Hygrophoraceae & Agaricales & 11. 11. $2017 \mathrm{~GB}$ \\
\hline Crepidotus mollis (Schaeff.) Stande & Inocybaceae & Agaricales & 25. 09. $2017 \mathrm{R}$ \\
\hline Crepidotus variabilis (Pers.) P. Kumm. & Inocybaceae & Agaricales & 10. 10. $2017 \mathrm{R}, \mathrm{DM}$ \\
\hline Inocybe rimosa (Bull.) P. Kumm. & Inocybaceae & Agaricales & $25.05 .2018 \mathrm{R}$ \\
\hline Marasmius candidus (Fr.) Singer & Marasmiaceae & Agaricales & 25. 07. $2018 \mathrm{~GB}, \mathrm{BR}$ \\
\hline Marasmius capillaris Morgan & Marasmiaceae & Agaricales & 26. 09. $2017 \mathrm{R}$ \\
\hline Marasmius oreades (Bolton) Fr. ** & Marasmiaceae & Agaricales & 20. 09. $2017 \mathrm{BA}$ \\
\hline Marasmius rotula (Scop.) Fr. & Marasmiaceae & Agaricales & 17. 06. $2018 \mathrm{R}, \mathrm{SH}, \mathrm{P}, \mathrm{BR}$ \\
\hline Megacollybia platyphyla (Pers.) Kotl. \& Pouzar & Marasmiaceae & Agaricales & 25.09. $2017 \mathrm{DM}$ \\
\hline Hemimycena pseudocrispula (Kühner) Singer & Mycenaceae & Agaricales & 11. 10. 2017. P \\
\hline Hemimycena tortuosa (P. D. Orton) Redhead & Mycenaceae & Agaricales & 01. 12. $2017 \mathrm{DM}$ \\
\hline Mycena filopes (Bull.) P. Kumm. & Mycenaceae & Agaricales & 30. 12. $2017 \mathrm{BR}$ \\
\hline
\end{tabular}


Table 1. continued

\begin{tabular}{|c|c|c|c|}
\hline Species & Family & Order & Date and place of collection \\
\hline Mycena galericulata (Scop.) Gray & Mycenaceae & Agaricales & 16. 10. $2017 \mathrm{DM}$ \\
\hline Mycena inclinata Fr. (Quél) & Mycenaceae & Agaricales & 25. 05. $2018 \mathrm{R}$ \\
\hline Mycena niveipes (Murrill) Murrill & Mycenaceae & Agaricales & 05. 07. $2018 \mathrm{CK}$ \\
\hline Mycena polygramma (Bull.) Gray & Mycenaceae & Agaricales & 25. 10. $2017 \mathrm{DM}$ \\
\hline Mycena pura (Pers.) P. Kumm. & Mycenaceae & Agaricales & 05. 07. $2018 \mathrm{CK}$ \\
\hline Mycena pura f. alba (Gillet) Kühner & Mycenaceae & Agaricales & 05. 05. $2018 \mathrm{~GB}$ \\
\hline Panellus stipticus (Bull.) P. Karst. & Mycenaceae & Agaricales & 20. 09. $2017 \mathrm{R}, \mathrm{SH}$ \\
\hline Gymnopus dryophilus (Bull.) Murrill & Omphalotaceae & Agaricales & 30. 12. $2017 \mathrm{R}, \mathrm{BR}$ \\
\hline Gymnopus foetidus (Sowerby) J. L. Mata \& R. H. Petersen & Omphalotaceae & Agaricales & 25. 09. $2017 \mathrm{R}$ \\
\hline Gymnopus peronatus (Bolton) Gray & Omphalotaceae & Agaricales & 02. 08. $2018 \mathrm{DM}, \mathrm{CK}$ \\
\hline Omphalotus illudens (Schwein.) Bresinsky \& Besl. & Omphalotaceae & Agaricales & 05. 01. $2018 \mathrm{~GB}$ \\
\hline Omphalotus olearius (DC.) Singer & Omphalotaceae & Agaricales & $\begin{array}{l}\text { 10. 07. } 2018 \mathrm{SH}, \mathrm{R}, \mathrm{BR}, \mathrm{K}, \\
\text { CK, P, DM }\end{array}$ \\
\hline Rhodocollybia butyracea (Bull.) Lennox & Omphalotaceae & Agaricales & 25. 07. $2018 \mathrm{~GB}$ \\
\hline Armillaria cepistipes Velen. & Physalacriaceae & Agaricales & 25. 10. $2017 \mathrm{DM}$ \\
\hline Armillaria gallica Marxm. \& Romagn. & Physalacriaceae & Agaricales & 25. 10. $2017 \mathrm{DM}$ \\
\hline Armillaria mellea (Vahl) P. Kumm. & Physalacriaceae & Agaricales & 13. 11. $2017 \mathrm{BA}$ \\
\hline Armillaria tabescens (Scop.) Emel & Physalacriaceae & Agaricales & 25. 10. $2017 \mathrm{DM}, \mathrm{R}$ \\
\hline Flammulina velutipes (Curtis) Singer & Physalacriaceae & Agaricales & 05. 01. $2018 \mathrm{~GB}, \mathrm{R}$ \\
\hline Hymenopellis radicata (Relhan) R. H. Petersen & Physalacriaceae & Agaricales & 11. 07. $2018 \mathrm{SH}$ \\
\hline Hohenbuehelia angustata (Berk.) Singer & Pleurotaceae & Agaricales & 02. 08. $2018 \mathrm{DM}$ \\
\hline Hohenbuehelia petaloides (Bull.) Schulzer & Pleurotaceae & Agaricales & 22. 07. $2018 \mathrm{SH}$ \\
\hline Pleurotus ostreatus (Jacq.) P. Kumm. & Pleurotaceae & Agaricales & 11. 10. $2017 \mathrm{P}$ \\
\hline Pluteus aurantiorugosus (Trog.) Sacc. & Pluteaceae & Agaricales & 10. 12. $2017 \mathrm{DM}$ \\
\hline Pluteus cervinus (Schaeff.) P. Kumm. & Pluteaceae & Agaricales & 10. 10. $2017 \mathrm{P}, \mathrm{SH}, \mathrm{R}$ \\
\hline Pluteus cervinus var. albus Peck. & Pluteaceae & Agaricales & 11. 07. $2018 \mathrm{SH}$ \\
\hline
\end{tabular}


Table 1. continued

\begin{tabular}{|c|c|c|c|}
\hline Species & Family & Order & Date and place of collection \\
\hline Pluteus nanus (Pers.) P. Kumm. & Pluteaceae & Agaricales & 16. 10. $2017 \mathrm{DM}$ \\
\hline Pluteus petasatus (Fr.) Gillet & Pluteaceae & Agaricales & 18. 07. $2018 \mathrm{SH}$ \\
\hline Volvariella bombycina (Schaeff.) Singer & Pluteaceae & Agaricales & 20. 09. $2018 \mathrm{BA}, \mathrm{SH}$ \\
\hline Volvopluteus gloiocephalus (DC.) Vizzini, Contu \& Justo & Pluteaceae & Agaricales & 30. 10. $2017 \mathrm{BA}, \mathrm{SH}, \mathrm{GB}$ \\
\hline $\begin{array}{l}\text { Coprinellus domesticus (Bolton) Vilgalys, Hopple \& Jacq. } \\
\text { Johnson }\end{array}$ & Psathyrellaceae & Agaricales & 22. 07. $2018 \mathrm{SH}$ \\
\hline Coprinellus micaceus (Bull.) Fr. & Psathyrellaceae & Agaricales & 25. 05. $2018 \mathrm{R}$ \\
\hline Coprinopsis lagopus (Fr.) Redhead, Vilgalys \& Moncalvo & Psathyrellaceae & Agaricales & 11. 10. $2017 \mathrm{P}$ \\
\hline Lacrymaria lacrymabunda (Bull.) Pat. & Psathyrellaceae & Agaricales & 10. 10. $2017 \mathrm{BA}, \mathrm{SH}$ \\
\hline Panaeolus papilionaceus (Bull.) Quél. & Psathyrellaceae & Agaricales & 11. $11.2017 \mathrm{~GB}$ \\
\hline Parasola auricoma (Pat.) Redhead, Vilgalys \& Hopple & Psathyrellaceae & Agaricales & 21. 05. $2018 \mathrm{BA}$ \\
\hline Parasola conopilus (Fr.) Örstadius \& E. Larss. & Psathyrellaceae & Agaricales & 17. 06. $2018 \mathrm{R}$ \\
\hline Psathyrella candolleana (Fr.) Maire & Psathyrellaceae & Agaricales & 25. 05. $2018 \mathrm{R}, \mathrm{CK}, \mathrm{P}$ \\
\hline Psathyrella pseudogracilis (Romagn.) M. M. Mosser & Psathyrellaceae & Agaricales & 10. 11.2017 BA \\
\hline Radulomyces confluens (Fr.) M. P. Christ. & Pterulaceae & Agaricales & 22. 07. $2018 \mathrm{SH}$ \\
\hline Schizophyllum commune Fr. & Schizophyllaceae & Agaricales & $\begin{array}{l}\text { 30.12.2017 BR, BA, R, } \\
\mathrm{P}, \mathrm{K}, \mathrm{DM}\end{array}$ \\
\hline Agrocybe molesta (Lasch) Singer & Strophariaceae & Agaricales & $09.09 .2017 \mathrm{BA}$ \\
\hline Hebeloma crustuliniforme (Bull.) Quél. & Strophariaceae & Agaricales & 10. 10. $2017 \mathrm{BA}$ \\
\hline Hypholoma fasciculare (Huds.) P. Kumm. & Strophariaceae & Agaricales & 16. 10. $2017 \mathrm{G}, \mathrm{P}, \mathrm{R}, \mathrm{SH}$ \\
\hline Hypholoma lateritium (Schaeff.) P. Kumm. & Strophariaceae & Agaricales & $25.09 .2017 \mathrm{R}$ \\
\hline Clitocybe gibba (Pers.) P. Kumm. & Tricholomataceae & Agaricales & 10. 07. $2018 \mathrm{SH}, \mathrm{CK}$ \\
\hline Clitocybe maxima (P. Gaertn., G. Mey. \& Scherb.) P. Kumm. & Tricholomataceae & Agaricales & 03. 08. $2018 \mathrm{DM}$ \\
\hline Clitocybe odora (Bull.) P. Kumm. & Tricholomataceae & Agaricales & 03. 08. $2018 \mathrm{SH}, \mathrm{DM}$ \\
\hline Melanoleuca brevipes (Bull.) Pat. & Tricholomataceae & Agaricales & 14. 11. $2017 \mathrm{~GB}$ \\
\hline Melanoleuca melaleuca (Pers.) Murrill & Tricholomataceae & Agaricales & 10. 12. $2018 \mathrm{DM}$ \\
\hline Phyllotopsis nidulans (Pers.) Singer & Tricholomataceae & Agaricales & 30. 12. $2017 \mathrm{BR}$ \\
\hline
\end{tabular}




\begin{tabular}{|c|c|c|c|}
\hline Species & Family & Order & Date and place of collection \\
\hline Pseudoclitocybe cyathiformis (Bull.) Singer & Tricholomataceae & Agaricales & 14. 11. $2017 \mathrm{~GB}$ \\
\hline Tubaria conspersa (Pers.) Fayod. & Tubariaceae & Agaricales & 05. 01. $2018 \mathrm{~GB}$ \\
\hline Tubaria furfuracea (Pers.) Gillet & Tubariaceae & Agaricales & 10. 03. $2018 \mathrm{BA}$ \\
\hline Athelia arachnoidea (Berk.) Jülich & Atheliaceae & Atheliales & 30. 12. $2017 \mathrm{BR}$ \\
\hline Athelia pyriformis (M. P. Christ.) Jülich & Atheliaceae & Atheliales & 20. 10. 2017 R, DM, SH \\
\hline Auricularia auricula-judae (Bull.) Quél. & Auriculariaceae & Auriculariales & 25. 10. $2017 \mathrm{DM}$ \\
\hline Auricularia mesenterica (Dicks.) Pers. & Auriculariaceae & Auriculariales & 05. 01. $2018 \mathrm{DM}, \mathrm{GB}$ \\
\hline Exidia glandulosa (Bull.) Fr. & Exidiaceae & Auriculariales & 17. 06. $2018 \mathrm{BR}, \mathrm{GB}, \mathrm{R}$ \\
\hline Exidia nigricans (With.) P. Roberts & Exidiaceae & Auriculariales & 30. 12. $2017 \mathrm{BR}, \mathrm{P}$ \\
\hline Exidia recisa (Ditmar) Fr. & Exidiaceae & Auriculariales & 30. 12. $2017 \mathrm{BR}$ \\
\hline Exidia thuretiana (Lév.) & Exidiaceae & Auriculariales & 30. 12. $2017 \mathrm{BR}$ \\
\hline Boletus subtomentosus J. A. Palmer & Boletaceae & Boletales & 11. 07. $2018 \mathrm{SH}$ \\
\hline Hortiboletus rubellus (Krombh.) Simonini, Vizzini \& Gelardi & Boletaceae & Boletales & 25. 05. $2018 \mathrm{R}$ \\
\hline $\begin{array}{l}\text { Imperator rhodopurpureus Assyov, Bellanger, Bertéa, Courtec., } \\
\text { Koller, Loizides, G. Marques, J. A Muñoz, N. Oppicelli, D. } \\
\text { Puddu, F. Rich. \& P. - A. Moreau }\end{array}$ & Boletaceae & Boletales & 03. 08. $2018 \mathrm{DM}$ \\
\hline Leccinum duriusculum (Schulzer ex Kalchbr.) Singer & Boletaceae & Boletales & 20. 06. $2018 \mathrm{BA}$ \\
\hline Neoboletus erythropus (Pers.) C. Hahn & Boletaceae & Boletales & 10. 07. $2018 \mathrm{SH}$ \\
\hline Xerocomellus chrysenteron (Bull.) Šutara & Boletaceae & Boletales & 10. 07. $2018 \mathrm{R}, \mathrm{SH}$ \\
\hline Xerocomellus porosporus (Imler ex Watling) Šutara & Boletaceae & Boletales & 10. 07. $2018 \mathrm{SH}$ \\
\hline Xerocomellus pruinatus (Fr. \& Höh) Šutara & Boletaceae & Boletales & 25. 05. $2018 \mathrm{R}$ \\
\hline Gyroporus castaneus (Bull.) Quél. & Gyroporaceae & Boletales & 11. 07. $2018 \mathrm{SH}$ \\
\hline Scleroderma citrinum Pers. & Sclerodermataceae & Boletales & 20. 05. $2018 \mathrm{BA}$ \\
\hline Scleroderma verrucosum (Bull.) Pers. & Sclerodermataceae & Boletales & 21. 07. $2018 \mathrm{R}, \mathrm{SH}$ \\
\hline Cantharellus cibarius Fr. ** & Cantharellaceae & Cantharellales & 10. 07. $2018 \mathrm{SH}, \mathrm{R}$ \\
\hline Craterellus cornucopiodes (L.) Pers. ** & Cantharellaceae & Cantharellales & 11. 07. $2018 \mathrm{SH}$ \\
\hline Dacrymyces capitatus Schwein. & Dacrymycetaceae & Dacrymycetales & 30. 12. $2017 \mathrm{BR}$ \\
\hline
\end{tabular}


Table 1. continued

\begin{tabular}{|c|c|c|c|}
\hline Species & Family & Order & Date and place of collection \\
\hline Geastrum fimbriatum Fr. & Geastraceae & Geastrales & 22. 07. $2018 \mathrm{~GB}$ \\
\hline Fomitiporia punctata (P. Karst.) Murrill & Hymenochaetaceae & Hymenochaetales & 16. 10. $2018 \mathrm{DM}$ \\
\hline Fuscoporia torulosa (Pers.) T. Wagner \& M. Fisch & Hymenochaetaceae & Hymenochaetales & 03. 04. $2018 \mathrm{DM}$ \\
\hline Hymenochaete rubiginosa (Dicks.) Lév. & Hymenochaetaceae & Hymenochaetales & 30. 12. $2017 \mathrm{BR}$ \\
\hline Inonotus dryadeus (Pers.) Murrill & Hymenochaetaceae & Hymenochaetales & 11. 10. $2017 \mathrm{P}$ \\
\hline Inonotus hispidus (Bull.) P. Karst. & Hymenochaetaceae & Hymenochaetales & 03. 05. $2018 \mathrm{BA}$ \\
\hline Phellinus igniarius (L.) Quél. & Hymenochaetaceae & Hymenochaetales & 30. 10. $2017 \mathrm{SH}$ \\
\hline Phellinus pomaceus (Pers.) Maire & Hymenochaetaceae & Hymenochaetales & 16. 10. $2017 \mathrm{DM}, \mathrm{BA}$ \\
\hline Phellinus robiniae (Murrill) A. Ames & Hymenochaetaceae & Hymenochaetales & 16. 10. $2017 \mathrm{G}$ \\
\hline Trichaptum biforme (Fr.) Ryvarden & incertae sedis & Hymenochaetales & 20. 10. $2017 \mathrm{P}, \mathrm{DM}$ \\
\hline Phallus hadriani Vent. * & Phallaceae & Phallales & 05. 07. 2018 GM, CK, BA \\
\hline Antrodia albida (Fr.) Donk. & Fomitopsidaceae & Polyporales & 20. 07. $2018 \mathrm{BA}, \mathrm{SH}$ \\
\hline Daedalea quercina (L.) Pers. & Fomitopsidaceae & Polyporales & 25.09. $2017 \mathrm{R}$ \\
\hline Laetiporus sulphureus (Bull.) Murrill & Fomitopsidaceae & Polyporales & 08. 09. $2017 \mathrm{BA}$ \\
\hline Ganoderma applanatum (Pers.) Pat. & Ganodermataceae & Polyporales & 17. 10. $2017 \mathrm{DM}$ \\
\hline Ganoderma lucidum (Curtis) P. Karst. & Ganodermataceae & Polyporales & 20. 05. 2016 DM, R, K, SH \\
\hline Ganoderma resinaceum Boud. & Ganodermataceae & Polyporales & 01. 10. $2017 \mathrm{~K}$ \\
\hline Meripilus giganteus (Pers.) P. Karst. & Meripilaceae & Polyporales & 25. 10. $2017 \mathrm{DM}$ \\
\hline Rigidoporus ulmarius (Sowerby) Imazeki & Meripilaceae & Polyporales & 20. 09. $2017 \mathrm{BR}$ \\
\hline Abortiporus biennis (Bull.) Singer & Meruliaceae & Polyporales & 25. 09. $2017 \mathrm{R}, \mathrm{CK}$ \\
\hline Bjerkandera adusta (Willd.) P. Karst. & Meruliaceae & Polyporales & 25. 09. $2017 \mathrm{~GB}$ \\
\hline Ceriporia spissa (Schwein. ex Fr.) Rajchenb. & Meruliaceae & Polyporales & 25. 10. $2017 \mathrm{DM}$ \\
\hline Cerocorticium molare (Chailler ex Fr.) Jülich \& Stalpers & Meruliaceae & Polyporales & 17. 06. $2018 \mathrm{BR}$ \\
\hline Gelatoporia dichroa (Fr.) Ginns. & Meruliaceae & Polyporales & 16. 10. $2017 \mathrm{BR}, \mathrm{P}$ \\
\hline Irpex lacteus (Fr.) Fr. & Meruliaceae & Polyporales & 25. 09. $2017 \mathrm{R}$ \\
\hline Phlebia radiata Fr. & Meruliaceae & Polyporales & 30. 12. $2017 \mathrm{BR}$ \\
\hline
\end{tabular}




\begin{tabular}{|c|c|c|c|}
\hline Species & Family & Order & Date and place of collection \\
\hline Phlebia tremellosa (Schrad.) Nakasone \& Burds. & Meruliaceae & Polyporales & 30. 12. $2017 \mathrm{BR}$ \\
\hline Steccherinum ochraceum (Pers.) Gray & Meruliaceae & Polyporales & 30. 12. $2017 \mathrm{BR}$ \\
\hline Byssomerulius corium (Pers.) Parmesto & Phanerochaetaceae & Polyporales & 30. 12. $2017 \mathrm{BR}$ \\
\hline Hapalopilus rutilans (Pers.) Murrill & Phanerochaetaceae & Polyporales & 28. 10. $2017 \mathrm{SH}, \mathrm{K}, \mathrm{P}$ \\
\hline Cerioporus squamosus (Huds.) Quél. & Polyporaceae & Polyporales & 25. 05. $2018 \mathrm{~GB}$ \\
\hline Cerrena unicolor (Bull.) Murrill & Polyporaceae & Polyporales & 25. 10. $2017 \mathrm{DM}$ \\
\hline Coriolopsis gallica (Fr.) Ryvarden & Polyporaceae & Polyporales & $25.09 .2017 \mathrm{R}$ \\
\hline Daedaleopsis tricolor (Bull.) Bondartsev \& Singer & Polyporaceae & Polyporales & 22. 07. $2018 \mathrm{SH}$ \\
\hline Daedaleopsis confragosa (Bolton) J. Schröt. & Polyporaceae & Polyporales & 25. 09. $2017 \mathrm{R}$ \\
\hline Fomes fomentarius (L.) Fr. & Polyporaceae & Polyporales & 25. 10. $2017 \mathrm{DM}, \mathrm{GB}$ \\
\hline Lentinus arcularius (Batsch.) Zmitr. & Polyporaceae & Polyporales & 13. $07.2017 \mathrm{R}$ \\
\hline Lentinus brumalis (Pers.) Zmitr. & Polyporaceae & Polyporales & $05.01 .2018 \mathrm{~GB}$ \\
\hline Lentinus tigrinus (Bull.) Fr. & Polyporaceae & Polyporales & $25.05 .2018 \mathrm{R}$ \\
\hline Pycnoporus cinnabarinus (Jacq.) P. Karst. & Polyporaceae & Polyporales & 11. 07. $2018 \mathrm{R}, \mathrm{SH}$ \\
\hline Trametes gibbosa (Pers.) Fr. & Polyporaceae & Polyporales & 05. 01. $2018 \mathrm{~GB}$ \\
\hline Trametes hirsuta (Wulfen) Lloyd & Polyporaceae & Polyporales & 13. 09. $2017 \mathrm{R}$ \\
\hline Trametes versicolor (L.) Lloyd. & Polyporaceae & Polyporales & 25. 09. $2017 \mathrm{R}, \mathrm{SH}, \mathrm{DM}$ \\
\hline Artomyces pyxidatus (Pers.) Jülich & Auriscalpiaceae & Russulales & 03. 01. $2018 \mathrm{~GB}$ \\
\hline Peniophora cinerea (Pers.) Cooke & Peniophoraceae & Russulales & 30. 12. $2017 \mathrm{BR}$ \\
\hline Peniophora quercina (Pers.) Cooke & Peniophoraceae & Russulales & 25. 10. $2017 \mathrm{R}$ \\
\hline Peniophora rufa (Fr.) Boidin & Peniophoraceae & Russulales & 01. 10. $2017 \mathrm{R}$ \\
\hline Lactarius fuliginosus var. albipes (J. E. Lange) & Russulaceae & Russulales & 25. 05. $2018 \mathrm{R}$ \\
\hline Lactarius fulvissimus Romagn. & Russulaceae & Russulales & 10. 07. $2018 \mathrm{SH}$ \\
\hline Lactarius quietus (Fr.) Fr. & Russulaceae & Russulales & 10. 07. $2018 \mathrm{SH}$ \\
\hline Russula amoenolens Romagn. & Russulaceae & Russulales & 10. 07. $2018 \mathrm{SH}$ \\
\hline Russula atropurpurea Peck. & Russulaceae & Russulales & 11. 07. $2018 \mathrm{SH}$ \\
\hline
\end{tabular}


Table 1. continued

\begin{tabular}{|c|c|c|c|}
\hline Species & Family & Order & Date and place of collection \\
\hline Russula cyanoxanthaf. peltereaui Singer & Russulaceae & Russulales & 11. 07. $2018 \mathrm{SH}$ \\
\hline Russula cyanoxantha (Schaeff.) Fr. $* *$ & Russulaceae & Russulales & 25. 05. $2018 \mathrm{R}$ \\
\hline Russula foetens Pers. & Russulaceae & Russulales & $25.05 .2018 \mathrm{R}$ \\
\hline Russula fragrans Romagn. & Russulaceae & Russulales & 10. 07. 2018 R, CK, SH \\
\hline Russula grata Britzelm & Russulaceae & Russulales & 05. 07. $2018 \mathrm{CK}$ \\
\hline Russula grisea Fr. & Russulaceae & Russulales & 10. 07. $2018 \mathrm{SH}, \mathrm{CK}$ \\
\hline Russula heterophyla (Fr.) Fr. & Russulaceae & Russulales & 25. 05. $2018 \mathrm{R}$ \\
\hline Russula nigricans Fr. & Russulaceae & Russulales & 22. 07. $2018 \mathrm{SH}$ \\
\hline Russula odorata Romagn. & Russulaceae & Russulales & 10. 07. $2018 \mathrm{SH}$ \\
\hline Russula rosea Pers. & Russulaceae & Russulales & 11. 07. $2018 \mathrm{SH}$ \\
\hline Russula rubroalba (Singer) Romagn. & Russulaceae & Russulales & 25. 05. $2018 \mathrm{SH}, \mathrm{R}$ \\
\hline Russula virescens (Schaeff.) Fr. ** & Russulaceae & Russulales & 11. 07. $2018 \mathrm{SH}, \mathrm{CK}$ \\
\hline Stereum hirsutum (Willd.) Pers. & Stereaceae & Russulales & 16. 10. $2017 \mathrm{P}, \mathrm{R}$ \\
\hline Stereum ochraceoflavum (Schwein.) Sacc. & Stereaceae & Russulales & 25. 10. $2017 \mathrm{R}$ \\
\hline Stereum ostrea (Blume \& T. Nees) Fr. & Stereaceae & Russulales & 01. 09. $2017 \mathrm{R}$ \\
\hline Stereum rugosum Pers. & Stereaceae & Russulales & 06. 10. $2017 \mathrm{R}$ \\
\hline Stereum subtomentosum Pouzar & Stereaceae & Russulales & 01. 10. $2017 \mathrm{R}, \mathrm{K}, \mathrm{P}$ \\
\hline \multicolumn{4}{|l|}{ ASCOMYCOTA } \\
\hline Hymenoscyphus albidus (Gillet) W. Phillips & Helotiaceae & Helotiales & 25. 10.2017 DM \\
\hline Hymenoscyphus epiphyllus (Pers.) Rehm ex Kauffman & Helotiaceae & Helotiales & 16. 10. $2017 \mathrm{P}$ \\
\hline Lachnum virgineum (Batsch.) P. Karst. & Hyaloscyphaceae & Helotiales & 05. 01. $2018 \mathrm{~GB}$ \\
\hline Helvella spadicea Schaeff. & Helvellaceae & Pezizales & 01. 05. $2018 \mathrm{BA}$ \\
\hline Peziza domiciliana Cooke & Pezizaceae & Pezizales & 06. 04. $2018 \mathrm{BA}$ \\
\hline Sarcoscypha coccinea (Jacq.) Sacc. & Sarcoscyphaceae & Pezizales & 15. 03. $2018 \mathrm{~GB}, \mathrm{R}$ \\
\hline Tuber aestivum (Wulfen) Spreng. ** & Tuberaceae & Pezizales & 13. 07. $2018 \mathrm{SH}, \mathrm{R}$ \\
\hline Xylaria hypoxylon (L.) Grev. & Xylariaceae & Xylariales & 11. 11. $2017 \mathrm{~GB}$ \\
\hline Xylaria polymorpha (Pers.) Grev. & Xylariaceae & Xylariales & 17. 06. $2018 \mathrm{~GB}, \mathrm{BR}, \mathrm{R}$ \\
\hline
\end{tabular}


The vast number of identified species of macromycetes in the territory of Batočina implicates that this area is suitable for their growth, despite the extensive destruction of habitats. This area was covered with dense forests in the past. Nowadays, many forests are destroyed and turned into building and agricultural land, while the remaining fragments of forests are surrounded by agricultural land and are under a huge impact of pesticides, used in agriculture. Macromycetes rarely grow in areas which are immensely under human impact and tend to withdraw to higher altitude areas, with limited human impact (RANKOVIĆ, 2014). In accordance with that, the biggest number of species of macromycetes was recorded on Straževica hill (the highest locality) and in Rogot (the only protected locality by the government).

According to the regulations of the Government of the Republic of Serbia (Sl. Glasnik RS", br. 5/2010 i 47/2011) there are several rare, protected and strictly protected species among the identified species. One of them (Phallus hadriani) is strictly protected (Fig. 2) and 7 are protected (Amanita caesarea, Marasmius oreades, Cantharellus cibarius, Craterellus cornucopiodes, Tuber aestivum, Russula cyanoxantha and $R$. virescens).

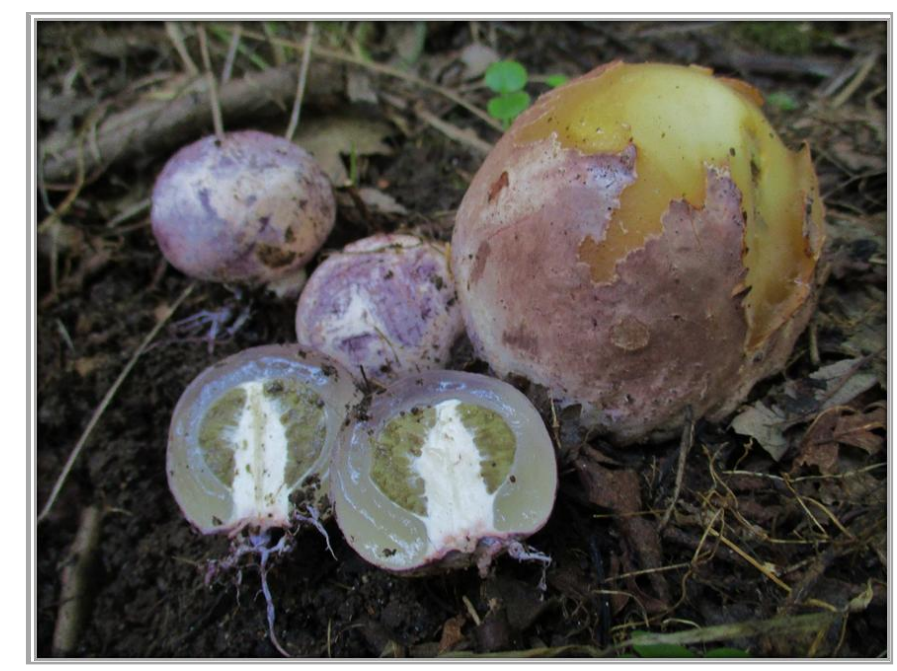

Figure 2. Strictly protected species Phallus hadriani Vent. (Photo: N. Petrović 05.07.2018)

Phallus hadriani, the dune stinkhorn, often grows in sandy soils, which aren't common in the territory of Serbia, thus this species is strictly protected. In Batočina municipality, this species was found in Gornja Mala, by the road in Batočina and in Crni Kao.

Amanita caesarea, Caesar's mushroom, is a mycorrhizal species, which often grows in thermophilic oak forests, or in forest edges. It is a highly regarded edible species, which has become endangered as a consequence to overharvesting and habitat destruction. It was found on Straževica hill only.

Marasmius oreades, the fairy ring mushroom, is an edible species, which grows in lawns and meadows. It was abundant in a meadow, by the road, in the town of Batočina.

Cantharellus cibarius, golden chanterelle, grows in broad-leaved and coniferous forests. It is edible and high-quality. It has become endangered due to overharvesting and habitat destruction. The presence of the golden chantarelle was noted in Straževica hill and Rogot.

Craterellus cornucopioides, the horn of plenty, is a species of edible mushroom, widely used because of its rich aroma. It often occurs in broad-leaved forests. It was noted in Straževica hill. Cantharellus cibarius and Craterellus cornucopioides are famous for 
accumulating cesium intensively, so mushroom gatherers should not collect these species on places rich with cesium (KOSTIAINEN, 2005).

Russula cyanoxantha, the charcoal burner, and $R$. virescens, the greencracked brittlegill, are edible and high-quality species, which is the reason why they are the most famous species of genus Russula among mushroom gatherers. They inhabit broad-leaved forests (FLIK, 2017). R. cyanoxantha was recorded in Donja Mala and Rogot and $R$. virescens was recorded in Straževica hill and Crni Kao.

According to literature (UZELAC, 2009) and sites (https://www.mushroomexpert.com/, http://www.gobe.si/, https://www.iucnredlist.org/) 11 species of the identified species are rare and endangered: Leucoagaricus americanus (Fig. 3), Coriolopsis gallica, Volvariella bombycina, Macrolepiota fuliginosa, Pluteus aurantiorugosus, Marasmius candidus, Phyllotopsis nidulans, Russula fragrans, $R$. grata, $R$. amoenolens and Helvella spadicea. $V$. bombycina, $R$. fragrans and $M$. candidus were frequent in the territory of Batočina.

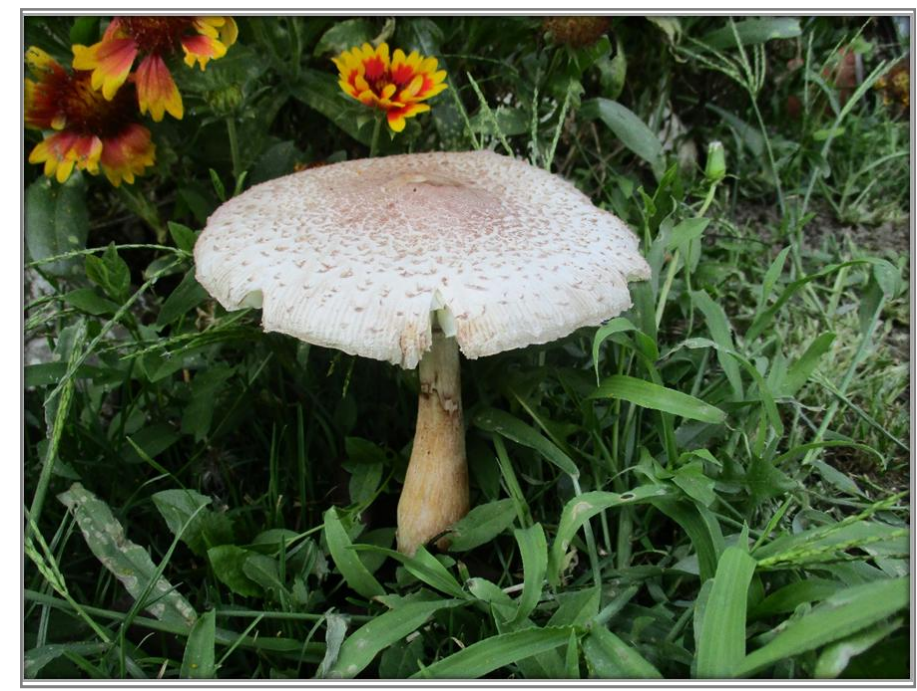

Figure 3. Rare species Leucoagaricus americanus (Peck.) Vellinga. (Photo: N. Petrović 28.07.2018)

While there aren't any published data on the diversity of macromycetes of Batočina, they exist for other areas of Serbia. Similar research on Kopaonik, in central Serbia, has shown the presence of 180 species of macromycetes in that area (IVANČEVIĆ, 1996). On Tara mountain, in western Serbia, 251 species of macromycetes were noted (CoLIĆ, 1967). On Bukulja mountain, in central Serbia, 225 species of macromycetes were recorded (LAČKOVIĆ, 2015). A total of 117 species of macromycetes was recorded on the Serbian side of Stara planina mountain, located in the eastern part of Serbia (IVANČEVIĆ and BERONJA, 2004). Apart from roughly comparing the number of recorded species of macromycetes in different areas, it is impossible to make any conclusions, since the vegetation and habitat conditions differ among investigated areas. Also, important factors are volume and duration of the research. The total number of species of macromycetes is certainly higher, but it is necessary to engage in research, far more extensive, covering the whole vegetation period over several years.

Researches of macromycetes diversity in various areas are crucial and mandatory for obtaining data of the number of species and their habitats, which is essential for preservation. 


\section{CONCLUSION}

Field studies on the diversity of macromycetes during 2017 and 2018 have shown that the area of Batočina is suitable for growth of many species of macromycetes. The presence of 200 species, which belong to phyla Basidiomycota and Ascomycota, was noted. Some of the identified species were strictly protected, protected and rare in the territory of Serbia.

Many localities remained unexplored or were explored in the wrong time, thus the total number of species might be even bigger, which is an opportunity for forthcoming researches.

This paper is a contribution to the knowledge of the diversity of macromycetes in the territory of Batočina and also a contribution to the knowledge of the diversity of macromycetes in Serbia.

\section{Acknowledgements}

This study was supported by the Ministry of Education, Science and Technological progress of the Republic of Serbia (project no. 173032)

\section{References:}

[1] ANONYMOUS (2010): Pravilnik o proglašenju i zaštiti strogo zaštićenih i zaštićenih divljih vrsta biljaka, životinja i gljiva ("Sl. Glasnik RS”, br. 5/2010). Accessed $10^{\text {th }}$ February 2019

[2] ANONYMOUS (2011): Pravilnik o proglašenju i zaštiti strogo zaštićenih i zaštićenih divljih vrsta biljaka, životinja i gljiva (“Sl. Glasnik RS”, br. 47/2011). Accessed $10^{\text {th }}$ February 2019

[3] Blackwell, M. (2011): The Fungi: 1, 2, 3... 5.1 million species? American Journal of Botany 98 (3): 426-438. doi: 10.3732/ajb.1000298.

[4] BoŽAC, R. (1984): Gljive - 600 gljiva naših krajeva. Mladost. Zagreb.

[5] Colić, D. (1967): Sinecological analysis of the fungal flora in a reserve with Serbia spruce (Picea omorica (Pančić) Purk.) on Mitrovac, Tara Mountain. Nature protection 34: 389-505. [in Serbian]

[6] FLIK, M. (2017): Koja je ovo gljiva? Edicija d.o.o. Beograd, Srbija.

[7] Focht, I. (1990): Ključ za gljive. ITP “Naprijed”. Zagreb, Hrvatska.

[8] Glavendekic, M., Kolarov, J. (1994): Fauna of Yugoslavian Ichneumonidae, Pimplinae, Xoridinae, Acaetininae (Insecta, Hymenoptera, Ichneumonidae). Zeitschrift für Entomologie 1: 1-12.

[9] HADŽIĆ, I. (2012): Priručnik za branje jestivih i lekovitih gljiva. Edicija. Beograd, Srbija.

[10] VuKoJEVIĆ, J., HADŽIĆ, I., (2013): Atlas gljiva i internacionalni rečnik narodnih imena gljiva. Biološki fakultet, Beograd, Srbija. 
[11] Hawksworth, D.L., LÜCKING, R. (2017): Fungal Diversity Revisited: 2.2 to 3.8 Million Species. Microbiology Spectrum 5(4). doi: 10.1128/microbiolspec.FUNK-00522016

[12] ILIĆ, M. (2014): Fizičko-geografske karakteristike opštine Batočina. Diplomski rad, Prirodno-matematički fakultet. Kosovska Mitrovica, Srbija.

[13] IVANČEVIĆ, B. (1996): Mycological research on the mountain of Kopaonik. Ekologija, 31(1): 45-53.

[14] IVANČEVIĆ, B., BERONJA, J. (2004): First records of macromycetes from the Serbian side of Stara Planina Mts (Balkan Range). Mycologia Balcanica 1(1): 15-19.

[15] IVANČEVIĆ, B. (2016): Prostorna distribucija i ekološke varijacije staništa hipogeičnih makromiceta (Mycota) u Srbiji. Doktorska disertacija, Biološki fakultet. Beograd.

[16] Kirk, P. M., Cannon, P. F., David, J. C., Stalpers, J. A. (2001): Ainsworth and Bisby's Dictionary of the Fungi (No. Ed. 9). CABI publishing. UK Centre, Egham, UK.

[17] Kostiainen, E. (2005): Household methods to reduce ${ }^{137}$ Cs contents of mushrooms. Radiological Protection in Transition. Proceedings of the 14. Regular Meeting of the Nordic Society for Radiation Protection, NSFS (No. SSI--2005-15).

[18] LAČKOVIĆ, N. (2015): Makromicete planine Bukulja i nizijskog područja Banje. Za$v r s ̌ n i ~ r a d$, Prirodno-matematički fakultet, Kragujevac, Srbija.

[19] Lukić, N. (2013): Rod Amanita u Srbiji. Gljivarsko društvo Šumadije, Kragujevac, Srbija.

[20] Marković, M., PaP, P., Drekić, M. Katanić, M. (2015): Mikološki kompleks na lišću i kori divlje trešnje (Prunus avium L.) u Republici Srbiji. Šumarstvo 1-2: 63-72.

[21] Moser, M. (1983): Keys to Agarics and Boleti: Polyporales, Boletales, Agaricales, Russulales. Mad River Pr Inc.

[22] Ranković, B. (2002): Gljive reda Erysiphales i njihovi paraziti u Srbiji. Prirodnomatematički fakultet, Kragujevac, Srbija.

[23] Ranković, B. (2014): Sistematika gljiva. Prirodno-matematički fakultet, Kragujevac, Srbija. SADIKOvić, D., KUŠTERA, M. (2013): Fungal conservation: Protected species of fungi in South Serbia region. Biologica Nyssana 4(1-2): 35-40.

[24] SAVIĆ, D. (2016): Diverzitet gljiva razdela Ascomycota na području Fruške gore sa posebnim osvrtom na red Helotiales. Doktorska disertacija, Prirodno-matematički fakultet. Novi Sad, Srbija.

[25] STAJIĆ, M. (2015): Nutritivna svojstva i medicinski potencijal makromiceta. Biološki fakultet, Beograd, Srbija.

[26] UzELAC, B. (2009): Gljive Srbije i zapadnog Balkana. BGV Logik. Beograd, Srbija. 
[27] Vukojević, J., Hadžić, I., KneŽević, A., Stajić, M., Milovanović, I., Ćilerdžić, J. (2016): Diversity of macromycetes in the Botanical Garden „Jevremovac" in Belgrade. Botanica Serbica 40(2): 249-259. doi: 10.5281/zenodo.162226

[28] https://nature.berkeley.edu/brunslab/ev/CHLOROPHYLLUM.pdf, $\quad$ Accessed $22^{\text {th }}$ February 2019

[29] http://www.gobe.si/ Accessed $13^{\text {th }}$ February 2019

[30] http://www.indexfungorum.org/names/names.asp Accessed $10^{\text {th }}$ February 2019

[31] https://www.iucnredlist.org/ Accessed $13^{\text {th }}$ February 2019

[32] https://www.mushroomexpert.com/ Accessed $13^{\text {th }}$ February 2019

[33] http://www.vielepilze.de/coprinus/copkey/ecopkey.pdf Accessed 22 ${ }^{\text {th }}$ February 2019

[34] http://www.zzps.rs/novo/kontent/stranicy/zastita_prirode_spomenici_prirode/Prirodni Spo menik.pdf Accessed $09^{\text {th }}$ February 2019 